\title{
Defining concepts of the digital society
}

\section{Christian Katzenbach}

The evolving digital society, Alexander von Humboldt Institute for Internet and Society, Berlin, Germany, christian.katzenbach@hiig.de

\section{Thomas Christian Bächle}

The evolving digital society, Alexander von Humboldt Institute for Internet and Society, Berlin, Germany, thomas.baechle@hiig.de

Published on 30 Nov 2019 | DOI: 10.14763/2019.4.1430

\begin{abstract}
At a time when branding new, occasionally innovative but often only catchy terms has become a familiar activity of researchers, companies and policymakers alike, it is necessary to reflect on which of these concepts is actually worthwhile, provides analytic value and in effect describes something new. This new special section Defining concepts of the digital society seeks to foster a platform that discusses and validates these overarching frameworks and theories. Based on the latest research, yet broad in scope, the contributions offer effective tools to analyse the digital society. Their authors offer concise articles that portray and critically discuss individual concepts such as algorithmic governance, datafication, platformisation, privacy with an interdisciplinary mindset. Each article contextualises their respective origin and academic traditions, analyses their contemporary usage in different research approaches and discusses their social, political, cultural, ethical or economic relevance and impact as well as their analytical value. The special section is a continuing project that will expand the collection of concepts in 2020 and beyond. We sincerely hope that it will grow into a valuable forum for making sense of the digital transformation and a pertinent resource for researchers, teachers, students and practitioners.
\end{abstract}

Keywords: Digital society

\section{Article information}

Published: 30 Nov 2019

Licence: Creative Commons Attribution 3.0 Germany

Competing interests: The author has declared that no competing interests exist that have influenced the text.

URL: http://policyreview.info/concepts/defining-concepts-digital-society

Citation: Katzenbach, C. \& Bächle, T. C. (2019). Defining concepts of the digital society. Internet Policy Review, 8(4). DOI: 10.14763/2019.4.1430 


\title{
FIRST CONCEPTS IN THIS COLLECTION
}

\section{Defining concepts of the digital society}

Christian Katzenbach \& Thomas Christian Bächle, Alexander von Humboldt Institute for Internet and Society

\author{
Algorithmic governance \\ Christian Katzenbach, Alexander von Humboldt Institute for Internet and Society \\ Lena Ulbricht, Berlin Social Science Center
}

\section{Datafication}

Ulises A. Mejias, State University of New York at Oswego

Nick Couldry, London School of Economics \& Political Science

\section{Filter bubble}

Axel Bruns, Queensland University of Technology

\section{Platformisation}

Thomas Poell, University of Amsterdam

David Nieborg, University of Toronto

José van Dijck, Utrecht University

\section{Privacy}

Tobias Matzner, University of Paderborn

Carsten Ochs, University of Kassel

\section{DEFINING CONCEPTS OF THE DIGITAL SOCIETY}

In our research on 'artificial intelligence', robots or autonomous systems in Berlin it is a recurring theme that preconceived images shape many of the expectations and fears associated with technologies. These images, however, do not necessarily reflect actual capabilities. Phenomena such as "machine learning" or "decision-making systems" are often misguidedly attributed with notions of intentionality, free will or consciousness. Still, these imaginations and figures of speech have actual political and social clout, shape research and technological development goals and inform discourses on regulation, innovation and potential futures.

Terminology shapes reality. What's true for the phenomena that we address in our research is certainly also true for the terminology we use for our research. What at first sounded like a banal truism, for us gradually evolved into the idea for this project, establishing a new special section Defining concepts of the digital society. At a time, when branding new, occasionally innovative but often only catchy terms has become a familiar activity of researchers, companies and policymakers alike, we felt it was particularly necessary to reflect on which of these concepts was actually worthwhile, provided analytic value and actually described something new besides the fluffy rhetoric that repeatedly becomes rampant in academic discourse.

Algorithmic governance, autonomous systems, transparency, smart technologies - these concepts are among the best candidates to serve this cause. They have become part of the vocabulary that is mobilised to make sense of the current rapid social and technological change. 
In this quest to understand the digital society, some ideas have proved to be more successful than others in stimulating public discourse, academic thinking, as well as economic and political activities. More recently, platformisation and datafication have become household-terms although relating to highly complicated and multi-facetted phenomena that could potentially also be described differently. Some concepts even strongly shape public and policy discourse albeit lacking solid empirical validation (the commonly referenced filter bubble is a case in point here).

There is high demand for concepts and explanations that condense the complexity of the world by transforming it into cogent and manageable ideas. Empirical research typically addresses single aspects of the current transformations. Adding small pieces to the puzzle, individual reports, research papers and essays tend to be rather unconnected, sometimes even resisting being combined with each other. While they certainly have a heuristic value, for example by validating or falsifying assumptions for well-defined, yet restricted contexts, they cannot provide overarching explanations and narratives. This is where more abstract concepts come into the picture. Operating on the level of middle range theories, they are able to integrate diverse phenomena under one notion by foregrounding certain shared characteristics. We need those overarching concepts to make sense of the current transformations.

\section{A NEW SPECIAL SECTION DEFINING CONCEPTS OF THE DIGITAL SOCIETY}

With this new special section Defining concepts of the digital society in Internet Policy Review, we seek to foster a platform that provides and validates exactly these overarching frameworks and theories. Based on the latest research, yet broad in scope, the contributions offer effective tools to analyse the digital society. Their authors offer concise articles that portray and critically discuss individual concepts with an interdisciplinary mindset. Each article contextualises their origin and academic traditions, analyses their contemporary usage in different research approaches and discusses their social, political, cultural, ethical or economic relevance and impact as well as their analytical value. With this, the authors are building bridges between the disciplines, between research and practice as well as between innovative explanations and their conceptual heritage.

We hope that this growing collection of reference papers will succeed in providing guidance for research and teaching as well as inform stakeholders in policy, business and civil society. For scholars, the articles seek to constitute an instructive reference that points to current research, historical and (interdisciplinary) backgrounds of the respective concepts, and relevant ongoing debates. For teachers and students alike, the articles offer an accessible overview that covers and contextualises broad themes while providing useful pointers to further research. Being relatively short and accessible in format, the articles thrive to become instructive and relevant beyond academia. Stakeholders in policy and business as well as journalists and civil society are increasingly interested in research evidence and academic perspectives on the entanglement of digitalisation and society. With its newly developed format the special section helps to navigate relevant research fields for these interdisciplinary questions. As an ongoing publication in this journal on internet regulation, we hope to not only meet the existing demand for overarching concepts and explanations but also being able to quickly adapt to the rapidly changing transformations.

\section{THE POLITICS OF CONCEPTS - AND THE LIMITS OF THIS SPECIAL SECTION}

Terms and concepts are lenses on the complexity of reality that foreground some aspects while neglecting others. They bear normative assumptions, install specific ways of understanding new 
phenomena, and possibly even create regulatory implications. The more we use these terms, the more both the phenomena they refer to as well as their specific framing increasingly become self-evident and ordinary. At the same time, however, each of these concepts has its own ideational, theoretical and rhetorical histories rooted, for example, in social theory or political thought, but also on a very practical level in business decisions to invest in certain ideas or policy debates with their own discursive rules. As a consequence, these concepts are far from being natural, let alone a neutral designator of existing phenomena. Concepts always bear their own politics - and in mobilising them, we need to carefully and critically reflect these politics and the choices they represent.

Of course, this special section on concepts of the digital society is necessarily and inescapably part of the very politics it seeks to reflect. By choosing certain terms over others, giving voice to a selection of authors, their respective disciplines and viewpoints, the special section itself undoubtedly takes part in the hierarchisation of terms and ideas. One could easily point at the limitations that result from providing predominantly Western perspectives, an uneven mix of disciplinary positions, even the dominant representation of certain auctorial subjectivities in terms of gender, race or ethnicity. Ultimately, any form of conceptual work struggles with blind spots. While we certainly acknowledge that the project poses challenges, we are certain that it is a worthwhile and necessary endeavour.

The special section is a continuing project. This first collection of five concepts offers a critical assessment of prominent, yet hitherto often nebulous or vague ideas, terms or descriptions. It does by no means seek to provide a finite and unalterable list of definitions. Its very objective is to encourage dialogue and contestation. We explicitly invite contributions to promote dialogue between the concepts and also to take counter-positions. With mostly co-authored pieces representing differing academic disciplines, the special section is already striving for a heterogeneity of viewpoints in individual papers. The larger quest of the project is to offer a genuine multitude of positions, extending, opposing or updating the concepts, their premises or consequences.

With this special section we are seeking to find a middle ground between the conceptual challenges and the aim of providing short and focused concept papers on the one hand and what we regard as the unquestionable need for interdisciplinary, concise and scholarly rigorous contributions that help to understand digital societies. This is the prime objective of this project.

\section{FIRST ARTICLES IN THE SPECIAL SECTION AND FUTURE CONCEPTS}

This launch of the special section in Internet Policy Review represents only the first installment of an ongoing project that seeks to build both a repertoire of instructive concepts and a platform to contest and elaborate on already published ones. Further iterations with additional concepts and commentaries on existing papers will follow in regular intervals.

With this first collection, the special section particularly focuses on the important role of data, the practices of their production, dissemination and trade as well as the ensuing broader social, political and cultural ramifications. Ulises A. Mejias and Nick Couldry look at the concept of datafication which describes a cultural logic of quantification and monetisation of human life through digital information. They identify the major social consequences which are aligned at the intersection of power and knowledge: in political economy, datafication has implications for labour and the establishment of new markets. Not only in this regard is it closely connected to the tendency - and concept - of platformisation (see below). With the help of decolonial theory Mejias and Couldry put particular emphasis on the politics and geography of datafication in 
what they call data colonialism: the large-scale extraction of data equals the appropriation of social ressources with the general objective (mostly by Western companies) to "dispossess". In the context of legal theory, Mejias and Couldry note that the processes of datafication are so wide-ranging that basic rights of the self, autonomy and privacy are increasingly called into question.

It is exactly this disposition of once authoritative ideas that has become quite fragile. In this context, Tobias Matzner and Carsten Ochs analyse the concept of privacy in relation to changing socio-technical conditions. They emphasise the need to understand and theorise privacy differently with the advent of digital technologies. These "shift the possibilities and boundaries of human perception and action" by creating visibilities and forms of interaction that are no longer defined by physical presence: personal information or pictures become potentially accessible for a worldwide audience, data "is easy and cheap to store" and becomes permanent in digital records. In addition to these technical contexts they argue that the scope of the "inherent individualism" of "conventional privacy theories" and data protection legislation does not meet the needs brought about by datafication: the forms of aggregated data used to identify behavioural patterns, they argue, is not the same as personal data.

One of the reasons why these forms of aggregated data operate at said intersection of knowledge and power is the practice of increasingly managing social spaces and interactions with algorithmic systems. Christian Katzenbach and Lena Ulbricht discuss algorithmic governance as a notion that builds on the longstanding theme that technology allows for a specific mode of governing society. Datafication, increasing computing power, more sophisticated algorithms, the economic and political interest in seemingly efficient and cost-reducing solutions, as well as the general trend towards digitalisation have all contributed to the new appeal and actual deployment of technological means to order the social. Eschewing the deterministic tendencies of the notion, yet taking seriously the increasing influence of algorithmic systems, the authors discuss a range of sectors from predictive policing to automated content moderation that increasingly rely on algorithmic governance. The concept brings previously unconnected objects of inquiry and research fields together and allows to identify overarching concerns such as surveillance, bias, agency, transparency and depoliticisation.

Many of these developments are primarily attributed to what we have converged on calling platforms: huge, often globally operating companies and services such as Facebook and Alibaba, Google and Uber that seek to transform and intermediate transactions across key economic sectors to position themselves as indispensable infrastructures of private and public life. Thomas Poell, David Nieborg and José van Dijck discuss platformisation as key development and narrative of the digital society. They argue that academic disciplines need to join forces in order to systematically investigate how changes in infrastructures, market relations and governance frameworks are intertwined, and how they take shape in relation to shifting cultural practices. We are only starting to understand how and why platforms have become the dominant mode of economic and social organisation and what the long-term effects might be.

One of the more prominent notions that seek to capture the effects of the reorganisation of social life by platforms and datafication is the metaphor of the filter bubble. Axel Bruns critically discusses this concept and carves out why it holds a special position in the set of concepts in this special section: while the idea of an algorithmically curated filter bubble seems plausible and enjoys considerable popularity in public and political discourse, empirical research shows little evidence that the phenomenon actually exists. Based on different readings of the concept and existing studies, Bruns argues that, rather than acutely capturing an empirical phenomenon, the 
persistent use of the notion has now created its own discursive reality that continues to have an impact on societal institutions, media and communication platforms as well as the users themselves. In consequence, the notion might even redirect scholarly attention away, warns Bruns, from far more critical questions such as why different groups in society "come to develop highly divergent personal readings of information" in the first place, and how the "ossification of these diverse ideological perspectives into partisan group identities" can be prevented or undone.

In 2020 the special section will continue, featuring concepts such as Digital commons, Transparency, Autonomous systems, Value in design and Smart technologies. Honouring the openness of the project, we appreciate suggestions for future concepts to be considered and any constructive feedback on the project itself. We sincerely hope the special section Defining concepts of the digital society will become a valuable forum and a helpful resource for many.

\section{Acknowledgments}

For an academic publication project such as this, most credit is routinely attributed to only a few named authors and editors. The success of a publication, however, always builds on a much broader group of people. This is particularly true for this special section. The long journey from the first idea to the publication of this collection of concepts was made possible by the help of many. We thank first and foremost Frédéric Dubois, the Internet Policy Review's managing editor who has steered this rocky ride from beginning to end, with careful attention, from the overarching process to the details of wording. Uta Meier-Hahn helped to push the idea towards realisation by drafting a first exposé. The board of the Internet Policy Review and colleagues gave valuable guidance, especially Melanie Dulong De Rosnay, Jeanette Hofmann, David Megías Jiménez, Joris van Hoboken, Seda Guerses and Lilian Edwards provided instrumental feedback on the way. And Patrick Urs Riechert gave all the manuscripts the final polish. Thank you all!

Thomas Christian Bächle and Christian Katzenbach

Berlin, December 2019 